Received 00th January 20xx Accepted 00th January 20xx DOI: $10.1039 / x 0 x \times 00000 x$

\title{
Study of Pseudomonas aeruginosa PPF-1 biofilm formation using microfluidics: effect of magnesium on biofilm detachment in engineered conduits
}

\author{
Jesse Greener, ${ }^{1,2 *}$ William Y. Harvey, ${ }^{3,4,5}$ Cynthia Gagné-Thivierge, ${ }^{3,4,5}$ Sepideh Fakhari, ${ }^{1}$ Jean \\ Barbeau $^{6}$ and Steve J. Charette ${ }^{3,4,5 *}$
}

\begin{abstract}
The bacterium Pseudomonas aeruginosa is an opportunistic pathogen in certain organisms, including humans, but can also survive and proliferate in natural and engineered water systems. Microfluidic technology can address hydrodynamic questions related to bacterial contamination of water flow systems and infrastructure. In this work, a microfluidic approach was devised to study the effect of shear stresses on biofilms from a dental unit waterline (DUWL)-isolated $P$. aeruginosa strain, PPF-1. During application of relevant shear stress levels to DUWLs, the response of the PPF-1 biofilm was observed and compared to a clinical $P$. aeruginosa reference strain, PAO1. The response measurements were repeated for biofilms exposed to additional $\mathrm{Mg}^{2+}$ ions. Using a microfluidic approach to transforming optical density maps into three-dimensional images, we applied computational fluid dynamics simulations and determined the critical shear stresses for biofilm sloughing. In the absence of $\mathrm{Mg}^{2+}$, PPF-1 biofilms showed weaker attachment than PAO1 biofilms, resulting in continuous slough/regrowth cycles triggered by applied shear stresses of $1.42+/-0.32 \mathrm{~Pa}$. Introducing $\mathrm{Mg}^{2+}$ into the PPF-1 biofilm culture medium seemed to place the biofilm into a viscoplastic mechanical state, thereby increasing mechanical stability, which resulted in elevated tolerances to shear stresses up to a critical value of $5.43+/-1.52 \mathrm{~Pa}$. This resulted in a propensity for less frequent but more catastrophic sloughing events like that observed for the PAO1 reference strain. This suggests that in a low ionic environment, biofilms from the PPF-1 strain can result in higher and more continuous ejection of biofilm materials, possibly leading to increased downstream colonization of engineered flow systems.
\end{abstract}

\section{Keywords}

Pseudomonas aeruginosa, biofilms, microfluidics, dental unit waterline, magnesium chloride, viscoplastic, shear stress, computational fluid dynamics.

\section{Introduction}

Biofilm proliferation in engineered water systems is an ongoing concern for both municipal water infrastructure and medical

${ }^{1}$ Département de chimie, Faculté des Sciences et de Génie, Université Laval, Québec, Québec, Canada, G1V OA6

${ }^{2}$ Centre de recherche du CHU de Québec, Université Laval, Québec, Québec, Canada, G1L $3 L 5$

${ }^{3}$ Institut de Biologie Intégrative et des Systèmes (IBIS), Université Laval, Québec, Québec, Canada, G1V OA6

${ }^{4}$ Département de biochimie, de microbiologie et de bio-informatique, Faculté des Sciences et de Génie, Université Laval, Québec, Québec, Canada, G1V OA6

${ }^{5}$ Centre de recherche de I'Institut universitaire de cardiologie et de pneumologie de Québec, Québec, Québec, Canada, G1V 4G5

${ }^{6}$ Département de stomatologie, Faculté de Médecine Dentaire, Université de Montréal, 2900 Edouard-Montpetit, Montréal, Québec, Canada, H3C 317

${ }^{*}$ Corresponding authors:

${ }^{1}$ Département de chimie, Faculté des Sciences et de Génie, Université Laval, Québec Québec Canada GIV OA6E-mail: jesse.greener@chm.ulaval.ca

${ }_{3}^{3}$ Institut de Biologie Intégrative et des Systèmes (IBIS), 1030, Avenue de la Médecine, Québec, Québec, Canada, G1V OA6 E-mail: steve.charette@bcm.ulaval.ca; equipment in healthcare environments. ${ }^{1,2,3}$ Research into those systems under flow conditions is especially important due to their prevalence and known effects of flow on biofilm mechanical properties and bacterial proliferation. 4,5 Pseudomonas aeruginosa is an opportunistic pathogen primarily known for its harmful role in the lungs of cystic fibrosis (CF) patients, ${ }^{6}$ but it also inhabits other environments, including human-made water installations. ${ }^{7,8}$ The $P$. aeruginosa PPF-1 strain was previously isolated from a dental unit waterline (DUWL) and is representative of other strains found in the same environment. ${ }^{9-11}$ Interestingly, similar to $P$. aeruginosa strains isolated from chronic CF lung infections, the gacS gene of PPF-1 is inactivated. ${ }^{11}$ This gene encodes a sensor kinase that is a component of a signalling network that regulates the expression of various virulence factors. ${ }^{12}$ PPF- 1 also contains a las $R$ gene alteration, ${ }^{11}$ as observed in other $P$. aeruginosa known to proliferate in engineered and natural water systems such as swimming pools, rivers and hydrocarbon-contaminated soils, hospital sink drains, and meat/fish market environments. ${ }^{13-15}$ The PPF-1 strain is an important producer of biofilms, ${ }^{9}$ and consequently, studying biofilm formation by PPF1 as a model strain is of interest. ${ }^{10}$ Biofilms are surface-attached structures made of cells embedded in a self-produced extracellular polymeric matrix. This helps to create a niche for cell growth and proliferation in otherwise inhospitable environments. ${ }^{11}$ As such, biofilm formation is likely the main 
strategy for efficient colonization of DUWLs and other engineered water systems. ${ }^{17}$

PPF-1 can form biofilms in static conditions and in pseudodynamic environments, such as within lightly shaken microplate cultures where bacteria exist under uncontrolled shear stress. ${ }^{10}$ The imposition of shear stresses produced by flow systems such as DUWLs may enhance biofilm downstream colonization through biofilm removal (sloughing) and control of oxygen availability. ${ }^{18}$ Flow systems also provide a supply of substrate and other molecules and avoid build-up of metabolic byproducts. To investigate flow-assisted biofilm sloughing, welldefined flow conditions should be applied while passive measurements are acquired. Amongst the numerous laboratory approaches available to study biofilms, ${ }^{19}$ the main tools (microtiter dish biofilm ${ }^{20}$ and Calgary Biofilm Devices ${ }^{21}$ ) provide a static growth environment and are therefore not useful for this study. Common flow reactors (drip flow reactors ${ }^{22}$ and the Center for Disease Control biofilm reactor ${ }^{23}$ ) do not provide a high degree of control over shear stresses, and other flow reactor types are not well adapted for long duration studies due to the large volumes of liquids required. ${ }^{24}$ To address these challenges, the biofilm research community is increasingly turning to microfluidic tools. ${ }^{25,26}$ In addition to reduced solution consumption, even for fast flow and long-duration experiments, the excellent optical properties of microfluidic fabrication materials enable straightforward transmission microscopy as a tool for high-quality time-lapse imaging. Due to their small dimensions, parallel flow microchannels, e.g., containing a sample and reference strain, can be monitored in the same field of view. Previously, we exploited these benefits to create threedimensional biofilm re-constructions and then used them to study the dynamic properties of Pseudomonas $s p$. biofilms in microchannels. ${ }^{27-29}$ This, combined with a complete lack of turbulence, results in highly predictable applied shear stress against wall-adhered biofilms, opening the way for computational fluidic dynamic simulations with realistic boundary conditions.

In a previous study, we demonstrated that in a shaken culture, divalent magnesium ions ( $\left.\mathrm{Mg}^{2+}\right)$ from added $\mathrm{MgCl}_{2}(20 \mathrm{mM})$ to the LB medium resulted in an increase in adhered biofilms. ${ }^{10} \mathrm{It}$ should be noted that LB medium typically contains only 0.05 to $0.3 \mathrm{mM}$ of $\mathrm{Mg}^{2+} .{ }^{30}$ The increased adherence was unique among the $P$. aeruginosa strains included in this study (PPF-1, PAO1, LESB58, and Urg-7). The effect of di- and trivalent ions on biofilm mechanical properties and their ability to resist sloughing from applied shear stresses are contradictory in the literature. ${ }^{31-33}$ Studies using microfluidics stand to clarify such discrepancies, especially, for example, contemporary questions related to the role of $\mathrm{Mg}^{2+}$ in biofilm accumulation and mechanical properties. ${ }^{34}$

In this work, we investigate the role of $\mathrm{Mg}^{2+}$ in stabilizing PPF-1 biofilm adhesion using new advances on previous microfluidic experiments and simulations studying biofilm mechanical properties. ${ }^{27-29}$ The results are compared with those from PPF1 biofilms under control conditions (no additional $\mathrm{Mg}^{2+}$ ). Comparisons to biofilms from the $P$. aeruginosa clinical strain PAO1 isolated from a human wound ${ }^{35}$ were used as a reference because they are well represented in a range of earlier microfluidic studies, ${ }^{36-39}$ albeit differently focused than the present research into the consequences of applied shear stress on biofilms. Specifically, we present quantitative results on critical shear stress for biofilm sloughing and cumulative biofilm release profiles over time. We also investigated the effect of $\mathrm{Mg}^{2+}$ ions, which caused an increased in mechanical stability and a propensity in PPF-1 for infrequent but catastrophic biofilm sloughing events, similar to the PAO1 reference strain. In contrast, PPF-1 that is not exposed to an increased $\mathrm{Mg}^{2+}$ ion concentration may more easily lead to downstream contamination.

\section{Materials and Methods}

\section{Flow cell fabrication and microfluidic setup}

The biofilms were grown inside microfluidic flow cells and were studied using a protocol adapted from a previous work. ${ }^{40}$ Using computer-aided design software (DraftSightTM, Dassault Systèmes, France), a flow cell containing two independent but closely spaced microchannels was designed to enable visualization of the sample and reference biofilms at the same time. A mould was fabricated using a dry (negative) photoresist film (Photopolymer Film 55, Mungolox, Germany) that was adhered to a glass slide with a heated laminator (304 Model, Fortex, UK). After lamination, the photoresist film was measured to have a thickness of $45 \mu \mathrm{m}$. This distance defined the channel height. The lateral dimensions of the microchannels were defined using a photomask, which was printed in-house using a high-resolution photoplotter (FPS 250000, Fortex, UK). A UV exposure system (UV-AY315, Fortex, UK) selectively crosslinked the photoresist through the photomask. After the unexposed photoresist was removed with a developer solution, the mould retained the features according to the design pattern. The flow cell was then made by casting a mixture of polydimethylsiloxane (PDMS) and a crosslinker against the mould. The PDMS device was subsequently sealed with a microscope glass coverslip by air plasma activation (PCD-001, Harrick Plasma, US). The final device featured two parallel microchannels with a height and width of 45 and $600 \mu \mathrm{m}$, respectively (Figure 1). A small inter-channel separation distance $(500 \mu \mathrm{m})$ enabled microscopy measurements of both channels in the same field of view while background light could be obtained from the space between them. Fluidic connections to the devices were made with perfluoro-alkoxy tubing (1514L, IDEX Corporation, Canada) at the entry and exit of each channel from the device PDMS side. Syringe pumps (SP300, Next Advance, USA) supplied a constant volumetric flow rate $(0.3$ $\left.\mathrm{mL} \cdot \mathrm{h}^{-1}\right)$ to the system. For each channel, a four-way diagonal flow valve (V-100D, IDEX Corporation, Canada) enabled a constant flow of liquid in the flow cell and air removal in the feed lines between syringe replacements. An upstream channel position was selected for observation to ensure contact with 
fresh liquid media and to prevent interference from upstream biofilm ejecta.

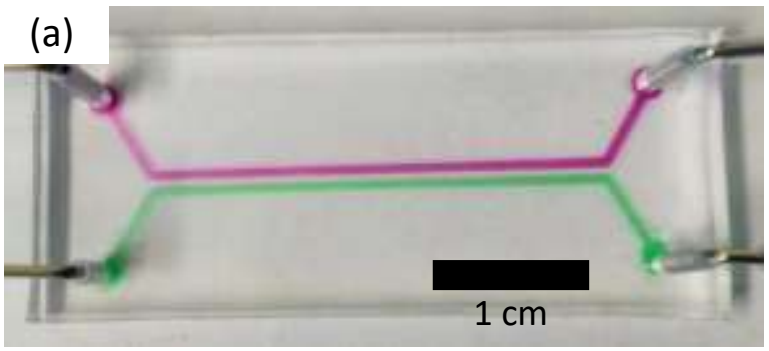

(b)

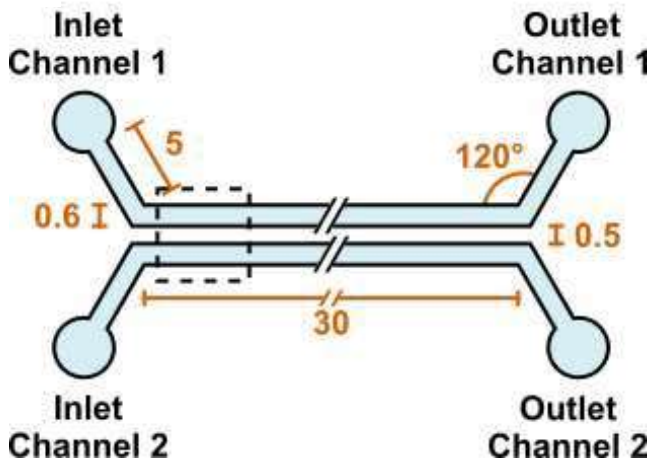

Figure 1. Microfluidic bioflow cell. (a) Photograph of the PDMS parallel-flow cell with the bonded microscope glass coverslip and coloured liquids to enhance the channel contrast. (b) Schematic view of the flow cell with measurements $(\mathrm{mm})$ of the two channels and the space between them. The dashed square indicates the microscope observation zone.

\section{Bacterial strains and pre-culture}

Two $P$. aeruginosa bacterial strains were used in this study: the PAO1 reference strain is a clinical isolate from a human wound, ${ }^{35}$ and the PPF-1 strain is a DUWL isolate, which is the focus of this study. ${ }^{9}$ From a single colony of bacteria grown overnight on tryptic soy agar (TSA, Wisent, St-Bruno, Canada), a bacteria pre-culture (room temperature, 200 revolutions per minutes, $25 \pm 5 \mathrm{~h}$ ) was prepared in $3 \mathrm{~mL}$ of $1 / 10$ diluted lysogeny broth (LB 1/10) (EMD, Mississauga, Canada) and used to inoculate the system.

\section{Biofilm formation in the microfluidic system}

A solution of $70 \%$ ethanol was injected into both device inlets at $0.35 \mathrm{~mL} \cdot \mathrm{h}^{-1}$ for 1 hour to disinfect the system. Sterile bidistilled water was then injected at $0.35 \mathrm{~mL} \cdot \mathrm{h}^{-1}$ for 1.5 hour to wash away any residual ethanol. The channels were inoculated by injecting the bacteria pre-culture at $0.25 \mathrm{~mL} \cdot \mathrm{h}^{-1}$ for 1.5 hour. Next, sterile LB $1 / 10$ medium was injected into the system at $0.30 \mathrm{~mL} \cdot \mathrm{h}^{-1}$. The biofilms were oxygenated due to both the high gas permeability of the PDMS and the natural oxygenation of the medium. For experiments assessing the effect of additional $\mathrm{Mg}^{2+}, 20 \mathrm{mM}$ of $\mathrm{MgCl}_{2}$ was added to the nutrient broth. LB 1/10 medium contains less than $0.03 \mathrm{mM}$ of $\mathrm{Mg}^{2+} .{ }^{30}$ All experiments were conducted at room temperature.

\section{Time-resolved imaging and analysis}

The flow cell was fixed atop an inverted microscope (LS620, Etaluma Inc., USA) with a $4 x$ objective for the duration of the experiment. Transmission images were automatically captured each hour, thereby creating a time-lapse image stack. The captured images were analysed using open source software (ImageJ, NIH, USA). ${ }^{41}$ The first image $(t=0)$ in each experiment was used as a background for calculating the $x-y$ biofilm optical density $(\mathrm{OD})$ maps, $\mathrm{OD}_{\mathrm{t}}(\mathrm{x}, \mathrm{y})$, according to:

$$
\underset{t}{O D}(x, y)=-\log \underset{I_{0}(x, y)}{\left(\frac{I_{t}(x, y)}{2}\right)}
$$

where $I_{t}$ is the pixel intensity at time $t, I_{0}$ is the pixel intensity at $t=0 h$, and the argument $(x, y)$ represents the horizontal and vertical pixel indices. To avoid inconsistencies associated with light flicker or changes to ambient light levels in the laboratory, the pixel intensities in all images were normalized to average background light levels obtained between the two microchannels where no biofilms existed. Following the creation of OD $x-y$ maps, the average OD value at a given time slice was obtained from $\mathrm{OD}_{\mathrm{t}}=\sum \mathrm{x}, \mathrm{y} \mathrm{OD}_{\mathrm{t}}(\mathrm{x}, \mathrm{y}) / \mathrm{n}$, where $\mathrm{n}$ is the number of pixels in the region of interest. This procedure has the effect of creating images on a black background with the pixel brightness corresponding to the quantity of biofilm present. ${ }^{42}$ The 3D biofilm structure was estimated using a previously validated calibration method to convert time-varying OD maps $\left(O D_{t}(x, y)\right)$ into biofilm topographical maps $\left(h_{b}, t(x, y)\right)$ at each pixel. ${ }^{40,43}$ Because the optical density to biofilm height calibration is applied individually to every experiment, it produces unique and self-consistent results for each experiment, irrespective of strain, growth conditions or even microchannel geometry. This approach was been shown to yield more reliable values for $h_{b}$ than direct measurements using confocal laser scanning microscope, especially for thin, earlygrowth-stage biofilms, ${ }^{43}$ or residual biofilm layers after sloughing, as in the present case. In this work, we used an improved multipoint calibration approach instead of the previous two-point method. See Electronic Supplementary Information for more details (Section S1).

Cumulative biofilm release and regrowth were calculated based on cumulative reductions and increases to average $O D$, respectively $\left(O D=\sum_{i, j} O D(i, j) / n\right.$, where $i$ and $j$ are pixel indices and $n$ is the total number of pixels being averaged). The coefficient of variance (CV) was calculated as a quantitative measure of the biofilm heterogeneity at any given time using:

$$
\mathrm{CV}_{\mathrm{t}}={ }^{\mathrm{OD}} \mathrm{\textrm {t }} / \mathrm{OD}_{\mathrm{t}} \times 100 \%
$$

where $\mathrm{OD}_{\mathrm{t}}^{\mathrm{STD}}$ is the standard deviation in $\mathrm{OD}_{\mathrm{t}}$.

\section{Flow simulations}

Computational fluid dynamic simulations were used to determine the average applied shear stress as a result of the 3D biofilm shape in the flow channel and the flow rate. Simulations (COMSOL Multiphysics 4.2a, Stockholm, Sweden) were run using three-dimensional biofilm models with the software 
option for "fine mesh" used with physics for laminar flow of an incompressible liquid. A series of control simulations were conducted to verify that the calculated shear stress results were independent of biofilm distribution across the upper and lower microchannel walls (Electronic Supplementary Information, Section S2).

\section{Results and discussion}

\section{Biofilm growth rates and structure}

In the first experiment, we compared the differences in the biofilm growth rate and structure of $P$. aeruginosa strains PPF1 and PAO1. Separate channels were inoculated the same time $(t=0)$ with an inoculum of either strain. After inoculation, a constant flow of LB 1/10 nutrient solution was passed through both channels. The volumetric flow rate of $0.3 \mathrm{~mL} \cdot \mathrm{h}^{-1}$ applied to the initially clean microchannel resulted in a shear stress of 0.37 $\mathrm{Pa}$ (Electronic Supplementary Information, Section S2). Imaging was conducted in the next 140 hour period to obtain time-lapse OD maps as biofilms of each strain grew in their respective channel. The full video of the PAO1 and PPF-1 biofilm accumulation and subsequent disruption events is available in the Electronic Supplementary Information (Video S1). Very little changed in the first $20 \mathrm{~h}$, corresponding to a lag phase in which the planktonic bacteria adapted to sessile life on the microchannel walls. After 20 hours, small, separated colonies were observable by microscopy. Initially, OD was nearly zero in both channels, while the $\mathrm{CV}$ values were large. Within just a few hours, the $\mathrm{CV}$ values for both channels were rapidly reduced as the colonies grew and merged. Both biofilms displayed similar

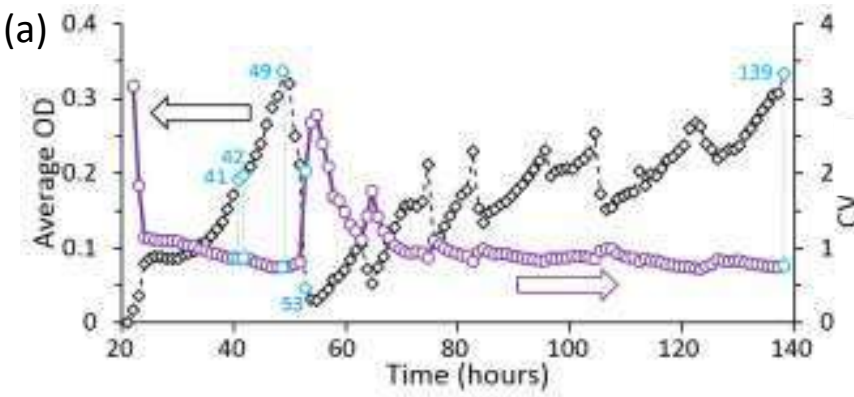

(b)

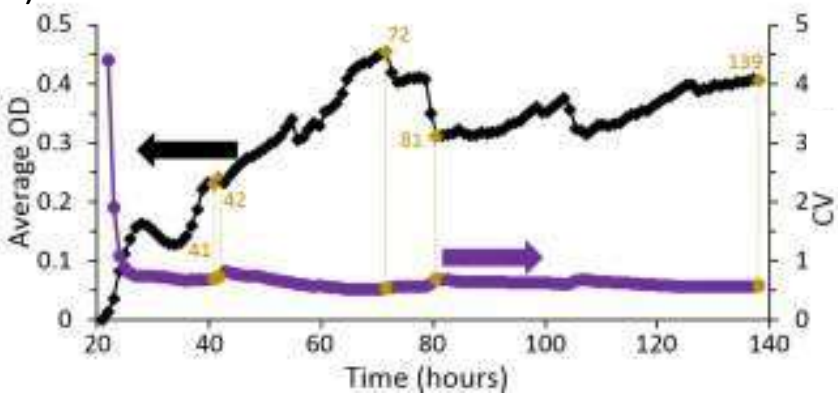

growth rates in the first 40 hours based on the average OD versus time curves (Figures $2 a$ and $2 b$ ). In the same time interval, significant differences in appearance were evident (Figure 2c). A textured surface was initially observed for the PAO1 strain, which is consistent with mushroom-like structures regularly described for PAO1 and other Pseudomonas strains. $^{36,44}$ After the initial formation of mushroom-like structures in PAO1, a process of reticulation occurred, which was clearly visible around 41 hours. This phenomenon was observed but was less obvious for the PPF-1 strain, which tended to produce a biofilm with a less textured topography.

\section{Biofilm displacement under shear flow}

Previous studies have shown that the adhesion strength of Pseudomonas biofilms can increase with applied shear stress, ${ }^{45,46}$ including for the PAO1 strain. ${ }^{47}$ Although this feature can improve adherence under temporary flow increases in engineered water systems, overgrowth of small conduits will eventually result in biofilm disruption due to the corresponding increases in shear stress. This process was observed after growth of both PPF-1 and PAO1 strains, with the initial effects presenting as a shifting-type displacement at approximately 41 hours in both cases. We define displacement as an abrupt movement of the biofilm, which nevertheless remains surface adhered. The changes in biofilm morphology due to displacement were initially more extensive for the PPF-1 strain, which demonstrated a significant corrugated structure afterwards. It appears that the PPF-1 may have only maintained contact with the walls via tethering to the channel while the biofilm largely lost contact with the horizontal channel surfaces.

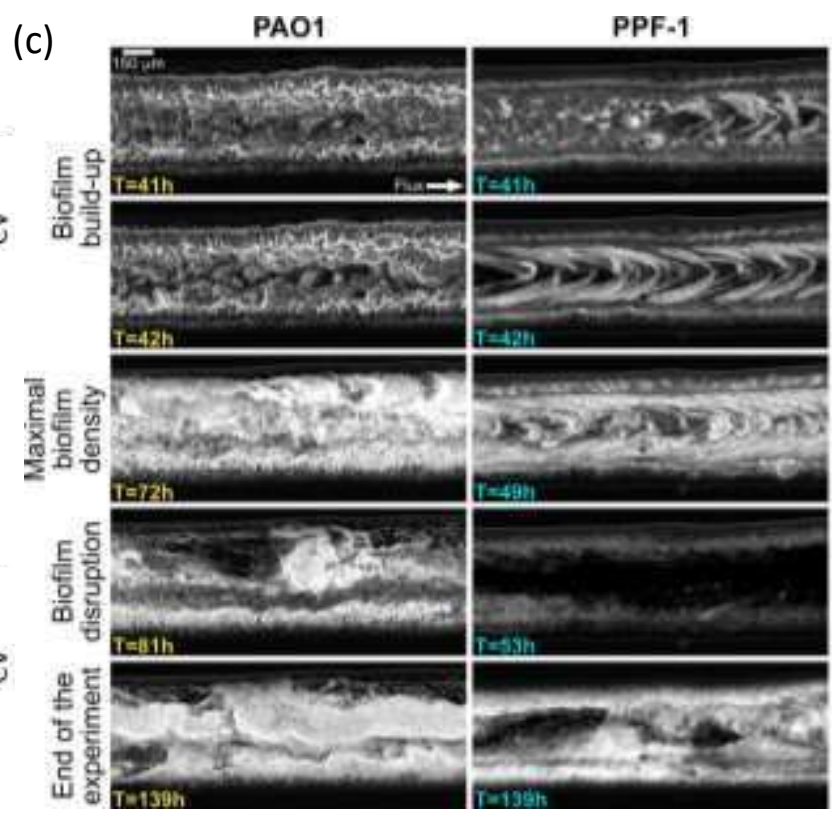

Figure 2. Comparison of results from time-lapse imaging of representative biofilms from PPF-1 and PAO1 strains of $P$. aeruginosa formed in a microfluidic bioflow cell exposed to $L B 1 / 10$ medium under continuous flow at $0.3 \mathrm{~mL} \cdot \mathrm{h}^{-1}$. Graph displaying average time-varying optical density (OD, black) and coefficient of variance (CV, purple) of the PPF-1 (a) and PAO1 (b) strains. Colour-coded arrows point to the relevant axis. The coloured dots in the graph highlight the key moments during the biofilm formation shown in (c). Prominent mushroom-like structures are shown in the PAO1 biofilm (highlighted at 41 and 42 hours). Corrugation in the PPF-1 strain is highlighted at the same times. Maximum biofilm density is highlighted at 72 and 49 hours, and the first biofilm sloughing events are finalized at 81 and 53 hours for PAO1 and PPF-1, respectively. Biofilms at the end of the experiment are shown at 139 hours. 
Two factors were likely responsible for the propensity for these displacement events after a certain time. The first is related to rapid changes to biofilm viscosity following the growth phase, ${ }^{40}$ which is especially pronounced in media solutions with high ionic strength, ${ }^{43}$ and this has been linked with changes to biofilm morphology via sudden partial detachment events. ${ }^{48}$ The second factor is a gradual increase in fluid velocity in the continuously decreasing head space above the growing biofilm, which increases the applied shear stress. ${ }^{49}$ After displacement, OD continued to increase, indicating the progression of biofilm overgrowth in the channel.

\section{Biofilm sloughing/re-growth cycles}

For the duration of the experiment, repeated slough/re-growth cycles in the PPF-1 strain were observed, as indicated by the continuous variations in the biofilm OD. Temporary increases in CV correlated with sloughing events indicating an increase in heterogeneity, likely due to a roughening of the exposed biofilm surface. After sloughing, the residual biofilm was largely contained in the microchannel corners where the applied shear stress was lower, and the larger local surface provided a higher density of anchor points. Following sloughing of the PPF-1 strain, regrowth occurred from the residual biofilm segments in the corners, causing a narrowing of the effective channel width and increases to the shear stress, as observed in other biofilm studies in low-aspect-ratio microchannels. ${ }^{40,49}$ In the case of the PAO1 strain, a two-stage sloughing event followed the maximum biofilm accumulation at 72 hours, and a less significant sloughing event occurred at $100 \mathrm{~h}$. It appeared that sloughing was less frequent for PAO1 than for PPF-1. Additionally, in contrast to PPF-1, sloughing played almost no role in modifying the heterogeneity of PAO1 (Figure 2b), indicating removal along a fracture boundary that resulted in no appreciable restructuring of the remaining biofilm. With temporary exceptions during and immediately following sloughing of the PPF-1 biofilm, the CVs of both biofilms generally tended toward lower values (more homogeneous) with time, but the PPF-1 strain was more heterogeneous than PAO1 throughout the experiment. Even after 140 hours, the CV of the PPF-1 was nearly twice that of the PAO1. Heterogeneous biofilm topographies are generally associated with higher exposed surface area, ${ }^{50}$ which is known to increase biofilm growth rates due to enhancements in nutrient loading. ${ }^{51,52}$ Because biofilms are known to present weaker mechanical properties (e.g., lower viscosity and Young's modulus) during fast-growth stages and to possess different cohesivity in the top versus base layers, ${ }^{53,54}$ it appears that the growth-slough cycles may produce a feedback loop that maintains the biofilm in a state that is conductive to pseudo-continuous production of ejecta into the flow stream.

\section{Critical shear stress}

Biofilm growth has a strong effect on the available channel free space and in turn can affect the local hydrodynamic conditions applied to the biofilm (specifically shear stress). We define the critical shear stress $\left(\tau_{c}\right)$ as the maximum shear stress experienced by the biofilm as it reached its critical volume, just before sloughing. To quantify $\tau_{c}$, we used a computational fluid dynamic model to simulate the shear stress based on a threedimensional representation of the biofilm shape in advance of each sloughing event. Estimates of the biofilm threedimensional shape were obtained as described in the Material and Methods section. In this way, we quantified the restrictions in channel free space as well as the corresponding changes in shear stress. As explained in the Electronic Supplementary Information (Section S3), the quantity of biofilm distributed on the top and bottom walls did not affect the calculated shear stress if the total biofilm volume was conserved along the channel cross-section. Therefore, for simplicity of visualisation and reduction of computational time, we represented the totality of the biofilm as adhered to the bottom wall. Visual representations of channels containing the PPF-1 or PAO1 strain at maximum overgrowth just before a sloughing event and the same channels immediately after sloughing are shown in Figure 3. In this case, the $\tau_{c}$ values generated due to flow of $0.3 \mathrm{~mL} \cdot \mathrm{h}^{-1}$ across the headspace, just before sloughing, were $\tau_{\mathrm{c}}=2.6$ and 6.1 Pa for PPF-1 and PAO1, respectively. The shear stresses applied to the residual surface adhered biofilms layers after the sloughing events shown in Figure 3 were 1.2 and 1.9 Pa for PPF1 and PAO1, respectively.

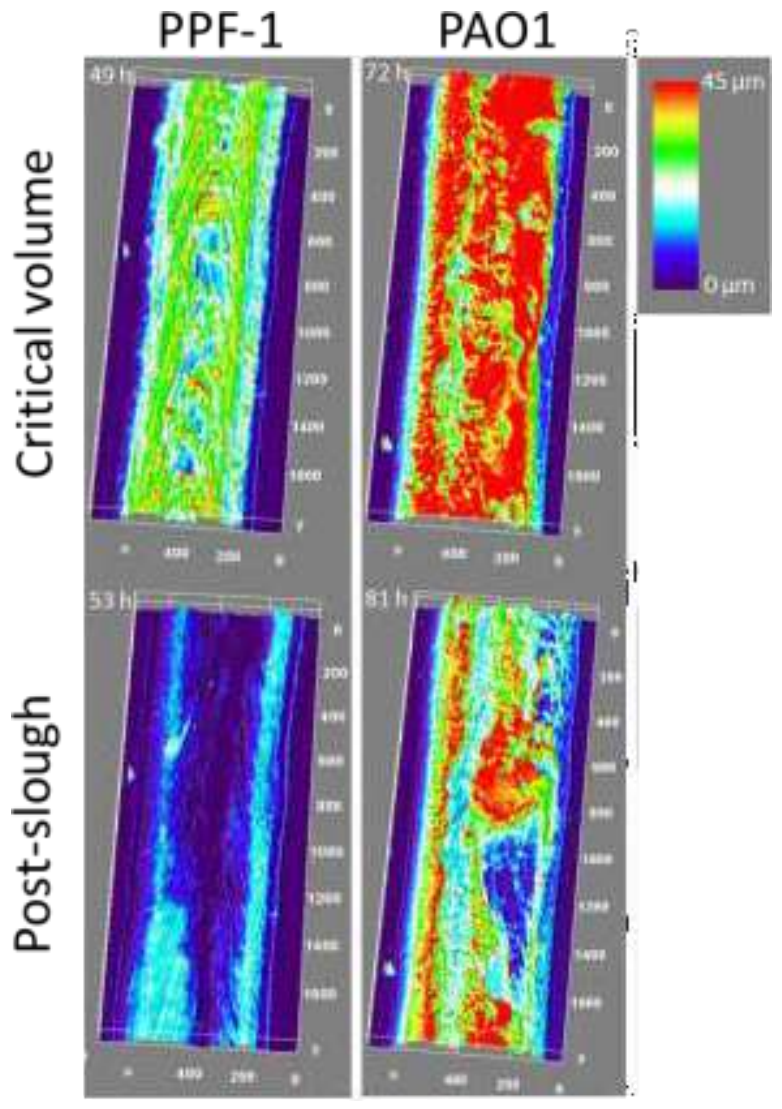

Figure 3. Three-dimensional rendering of PPF-1 (left) and PAO1 (right) biofilms at their critical volumes (top) and after sloughing (bottom) for the experiments shown in Figure 2. Times are indicated in the top left corner for each rendering. Distances along the $x$ - 
and $y$-axes are shown in units of $\mu \mathrm{m}$. Colour bar associates false colour scale to height. Flow is from bottom to top in all images.

We conclude that biofilms from the PPF-1 strain are more likely to slough than those from the PAO1 strain. This is the result of three characteristics of the PPF-1 biofilm: (i) lower attachment strength to the microchannel, (ii) rapid and nearly continuous regeneration of new growth biofilms, and (iii) lower $\tau_{c}$ required for sloughing. Each factor is linked to the biofilm mechanical properties and is evidenced by the higher number of sloughing events observed for PPF-1 compared to PAO1.

\section{Stiffening of PPF-1 biofilm at a high concentration of magnesium salt}

Based on previous studies reporting that biofilms from PPF-1 exposed to $\mathrm{Mg}^{2+}$ in a shaken culture resulted in an increase in adhered biofilms, we chose to study the impact of $\mathrm{Mg}^{2+}$ ions on biofilms under dynamic flow conditions. The experiment was repeated four times with PPF-1 exposed to $\mathrm{Mg}^{2+}$ and under control conditions in the reference channel (no $\mathrm{Mg}^{2+}$ addition). Due to the differences in the OD versus time profiles, including differential effects from the lag phase, growth rate and timing of biofilm sloughing events, the results from four individual experiments for each condition are plotted in Figures 4a rather than their mean results. Despite the variability in these results, certain observations are possible. First, in the presence of $\mathrm{Mg}^{2+}$ ions, biofilms accumulated into more optically dense and thicker layers while also demonstrating greater resistance to shear stress and detachment compared to those under control conditions. This is shown in Electronic Supplementary Information, Section S3 for each individual experiment via $h_{b}$ and the cumulative release profiles versus time. Second, preceding the detachment, displacement events occurred that resulted in corrugated topographical structures, as observed previously under control conditions. Such displacements preceded sloughing, but continued nearly continuously between sloughing events for low-ionic-strength nutrient solutions, compared to those with added $\mathrm{Mg}^{2+}$, but never showed signs of catastrophic by sloughing ripping (Figure 4c). Based on the increases to the PPF-1 biofilm surface attachment, stiffness, and accumulated material before reaching the critical volume strain in the presence of $\mathrm{Mg}^{2+}$ ions, we conclude that the biofilm more closely resembled PAO1 than PPF-1 under the control conditions. Third, after reaching a critical volume (and corresponding $\tau_{c}$ ), the $\mathrm{Mg}^{2+}$-exposed biofilms sloughed off abruptly and massively via a tearing process (Figure $4 \mathrm{~b}$ ). The clean edges at the fracture boundary indicate that the biofilm had become brittle. This evidence points to a viscoplastic deformation behaviour (e.g., as described by Binham and Herschel-Buckley models). Generally, biofilms are treated as viscoelastic materials, which can respond to both long-term shear stresses by viscous flow and to short-term perturbations by a reversible elastic response. However, the concept of biofilm plasticity is a new idea, one by which large internal stresses are irreversibly relieved. ${ }^{54,55}$ In this case, biofilm plasticity can be viewed as the irreversible deformation that occurs by ripping at the stress fracture boundaries as a response to large shear stresses applied to a highly viscous state obtained by the mature biofilm when it is exposed to a high-ionicstrength nutrient. ${ }^{43}$ Therefore, this observation may help to accelerate further inquiry, including the creation of predictive models for critical shear stress based on viscoelastic parameters. This type of deformation, which we call catastrophic sloughing, was observed in 3 out of 4 experiments using added $\mathrm{MgCl}_{2}$. In the one experiment in which the sloughing was not observed at all, the biofilm height was only $32.2 \mu \mathrm{m}$ by the end of the experiment, whereas in the other 3 experiments, the average biofilm height was significantly higher $\left(h_{b}=39.1 \pm 3.0 \mu \mathrm{m}\right)$. Therefore, it seems likely that a critical biofilm volume had not been reached before the end of the 68 hours in the experiment in which sloughing was not observed.

\section{Cumulative release}

The accumulated difference in the height-calibrated OD before and after each sloughing event was used to calculate the cumulative biofilm loss into the effluent (Figure 4d). This view shows that in the control experiments (no $\mathrm{Mg}^{2+}$ addition), release of biofilm segments into the effluent phase started approximately 10 hours before that of $\mathrm{Mg}^{2+}$-exposed biofilms. As well, during the control experiments, the biofilm release occurred continuously, displaying a continuous periodic sloughing mode, which we refer to as 'pseudo-continuous sloughing', whereas the $\mathrm{Mg}^{2+}$-exposed biofilms followed a stepwise release pattern resulting from singular catastrophic sloughing events. Interestingly, by the end of the experiment, the cumulative release of control and $\mathrm{Mg}^{2+}$-exposed biofilms were statistically the same. Nevertheless, it is expected that the liberated biofilm segments from the $\mathrm{Mg}^{2+}$-crosslinked biofilm would not be as efficient in downstream colonization as the control samples because most bacteria would be contained within dense and highly crosslinked ejecta with relatively low surface-area-to-volume ratios. In addition, large solid biofilm segments present the opportunity for filtration, whereas smaller deformable biofilm segments from control experiments may pass through filters to access and colonize downstream conduits. These results suggest that the application of divalent solutions coupled with high flow conditions could result in shear removal and filtration of large biofilm as a potential route to better decontamination. This goal could be achieved without aggressive antibacterial agents by provoking catastrophic sloughing after $\mathrm{Mg}^{2+}$ exposure or by applying antibacterial treatments after $\mathrm{Mg}^{2+}$-aided catastrophic sloughing to limit or prevent regrowth from exposed residual layers. 
(a) 0.7$]=-[B-1 h 0$

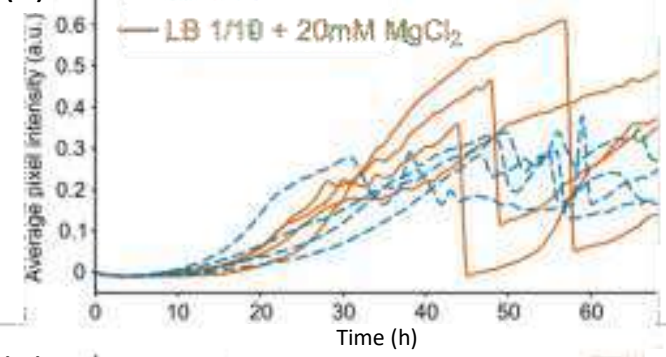

(d) $0.6-=-L B 1 / 10$

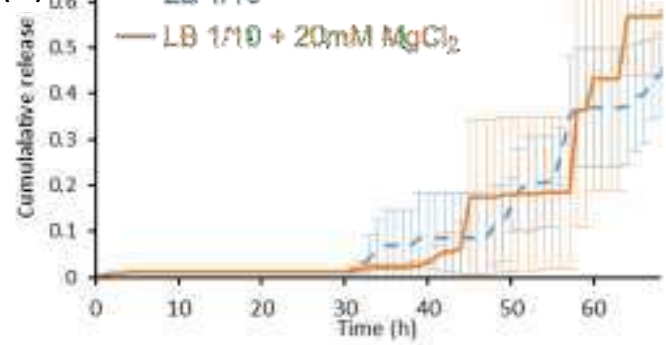

(b)

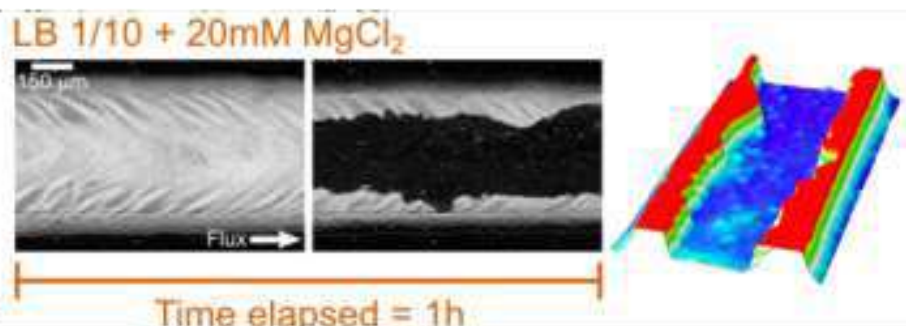

(c)

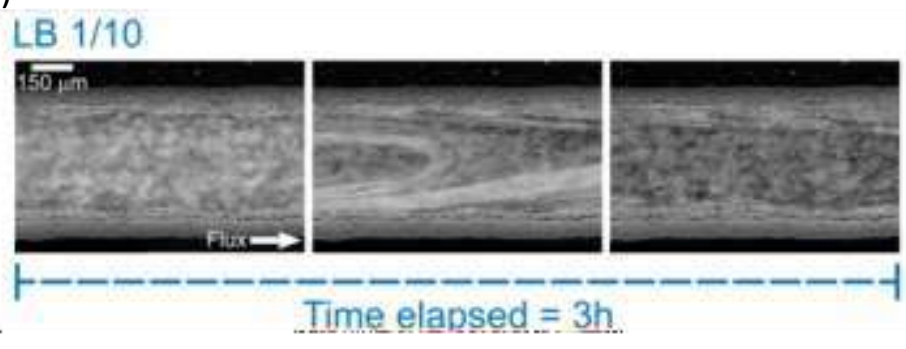

Figure 4. Effect of $\mathrm{MgCl}_{2}$ on biofilm formation of the PPF-1 strain of $P$. aeruginosa. (a) Time series of optical density measurements for PPF-1 biofilms under $0.3 \mathrm{~mL} \cdot \mathrm{h}^{-1}$ flow of $\mathrm{LB} 1 / 10$ medium supplemented with $20 \mathrm{mM}$ of $\mathrm{MgCl}_{2}$ (orange) or control conditions without $\mathrm{MgCl}_{2}$ addition (blue dashed) (a.u. = arbitrary units). (b) Average cumulative release profile data in (a). Colour coding is the same in (a) and (b). Error bars in (b) are the result of the standard deviation from cumulative release ( $n=4)$. Representative results from sloughing events for $(c) \mathrm{MgCl}_{2}$-exposed samples and for (d) control experiments. Subfigures are labelled in the order that they appear in the main text, but (d) is placed under (a) for better comparison between OD and cumulative release time profiles.

We conclude this study with a comparison of critical shear stress values for the three experimental conditions used in this paper. This was accomplished by simulations using the threedimensional reconstructed biofilm shape to calculate the shear stress $\left(\tau_{c}\right)$ immediately before the sloughing events, and shear stress after sloughing was determined in the same manner. Average $\tau_{c}$ values were obtained from multiple sloughing events recorded during time-lapse videos from each experiment to obtain statistical significance. We also compared the average $\tau_{c}$ values with the shear stress values after sloughing to determine how significantly sloughing affected the hydrodynamic conditions in the vicinity of the biofilm. Data for PPF-1 in the control condition (no $\mathrm{Mg}^{2+}$ addition) were averaged over 4 separate experiments, yielding a critical shear stress value of $\tau_{c}$ $=1.42+/-0.32 \mathrm{~Pa}$. Due to the relatively insignificant effect of the pseudo-continuous sloughing events on the remaining biofilm volume, the post-sloughing shear stresses $(0.95+/-0.27$ $\mathrm{Pa}$ ) were not statistically different from $\tau_{\mathrm{c}}$. It can be concluded that the slough/regrowth states became intertwined after the sloughing started, leading to a pseudo-continuous biofilm ejection. Interestingly, in a previous study, a similar observation was made for the release of planktonic cells from Pseudomonas $s p$. biofilms in a microchannel at subcritical shear stresses by counting CFU from effluent. ${ }^{56}$ In comparison, the addition of 20 $\mathrm{mM} \mathrm{MgCl}$ to the nutrient solution (LB $1 / 10$ medium) dramatically increased the PPF-1 resilience to sloughing, enabling the biofilm to continue to grow until critical shear stress values of $\tau_{c}=5.43+/-1.52$ Pa were applied. As a reminder, the LB $1 / 10$ medium used in this study contains less than 0.03 $\mathrm{mM}$ of $\mathrm{Mg}^{2+} .30$ The stronger cohesion that enhanced resistance to shear stress also resulted in the tendency for the selfcohesive and brittle biofilm to rip from the channel in a large single ejecta via a similar viscoplastic deformation observed for
PAO1 biofilms. The large loss of biofilm led to a significant reduction in applied shear stress after sloughing of $\tau_{c}=0.50+/$ $0.38 \mathrm{~Pa}$, after which the biofilm began to grow again. Thus, the addition of $\mathrm{MgCl}_{2}$ causes the sloughing and regrowth phases to become separate and distinct. This behaviour was similar to that of the PAO1 reference strain, which experienced distinct $\tau_{c}$ and post-sloughing shear stresses of $4.23+/-1.22 \mathrm{~Pa}$ and 1.67 +/- $0.22 \mathrm{~Pa}$, respectively. We also note that elevated critical shear stress values correlated to larger slough volumes during any singular event. However, it should be reiterated that the total cumulative detached biofilm volume involving PPF-1 strains under different ionic concentrations was similar despite the significant differences in the biofilm volume released per sloughing event. Figure 5 also estimates the average biofilm volume lost during a sloughing event for PPF-1 under control conditions, under $\mathrm{MgCl}_{2}$ exposure, and for the $\mathrm{PAO} 1$ reference experiments. As expected, the volume lost during a single sloughing event was largest for PPF-1 exposed to $\mathrm{Mg}^{2+}$.

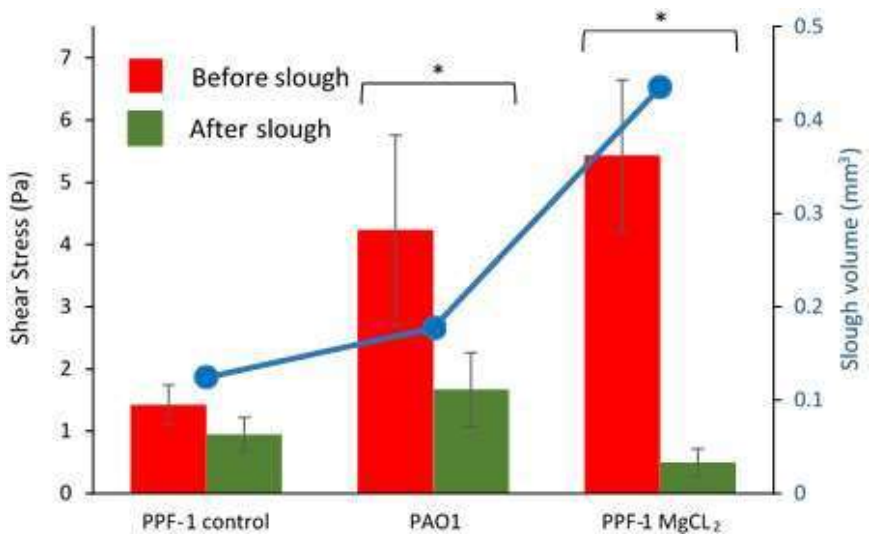

Figure 5. Average shear stresses before and after sloughing events (red and green, respectively). Error bars show the standard deviation in the average measurements. 
Measurements were the result of multiple sloughing events during each of the experiments. In total, 5 experiments were run using PPF-1 control (no $\mathrm{Mg}^{2+}$ addition), 4 experiments using PPF-1 with $20 \mathrm{mM} \mathrm{MgCl}$ added and 1 experiment using PAO1. Average volume $\left(\mathrm{mm}^{3}\right)$ of biofilm removed from the microchannel during each sloughing event is shown in blue. Statistical significance in shear stress values before and after sloughing events from a pairwise t-test is indicated by*.

\section{Conclusion}

Strains of PPF-1 from Pseudomonas aeruginosa isolated from dental unit water lines were studied in microchannels using time-lapse imaging, and the results were compared to those from a reference strain of PAO1. Using height-calibrated OD maps, three-dimensional biofilm topographies were created, and the applied shear stresses were modelled using computational fluid dynamics simulations. While marked differences existed between biofilms from PPF-1 and the reference PAO1, PPF-1 exposure to $\mathrm{Mg}^{2+}$ ions changed its mechanical properties and became similar to those of the reference strain. These properties included an increased ability to resist sloughing until the applied shear stresses surpassed a critical value. The resulting catastrophic sloughing showed evidence of viscoplastic failure, an understudied mechanical regime in the biofilm literature. The extreme differences in the $\mathrm{Mg}^{2+}$-exposed PPF-1 biofilm shape after sloughing points to highly distinctive sloughing and post-sloughing growth states. Conversely, in a low-ionic-strength environment, shear stresses applied before and after PPF-1 biofilm sloughing were not significantly different. For the latter case, this indicates that the sloughing and post-sloughing growth states were highly intertwined, which can result in a nearly continuous process of bacterial ejection and downstream colonization. Therefore, $\mathrm{Mg}^{2+}$ addition could play a role in future strategies for limiting Pseudomonas aeruginosa contamination of dental unit water lines and other engineered water systems.

\section{Author Contributions}

JG: formal analysis, methodology, supervision, visualization, writing; WYH: investigation, writing; CG-T: investigation, writing, methodology, formal analysis; SF: software, formal analysis; JB writing, conceptualization; SJC: conceptualization, methodology, supervision, resources, writing.

\section{Conflicts of interest}

There are no conflicts to declare.

\section{Acknowledgements}

The authors thank Aimee Dawson for discussions about dental unit water lines, Mohammad S. Taghavi for discussions regarding mechanical properties of complex materials and biofilms, Molly K. Gregas for technical edits to the manuscript, Luc Trudel for his help with calculations on trace ion concentrations, and for help with device fabrication and photography thanks goes to Jayesh Sonawane, Nastaran
Khodaparastasgarabad, Nan Jia, for help with photography and device fabrication. Authors also thank funders for their support. These include the Institut universitaire de cardiologie et de pneumologie de Québec as a scholarship to WYH and by the Natural Sciences and Engineering Research Council of Canada [RGPIN-2019-04444] to SJC and [RGPIN-2020-03603] to JG. SJC is a research scholar of the Fonds de Recherche du QuébecSanté.

\section{References}

1 K.E. Fish, A.M. Osborn, J. Boxall, Environ Sci Water Res Technol, 2016, 2, 614-630.

2 A. Mahapatra, N. Padhi, D. Mahapatra, M. Bhatt, D. Sahoo, S. Jena, D. Dash, N. Chayani, J Clin Diagn Res. 2015, 9, DC09DC11.

3 R. M. Donlan, Emerg Infect Dis, 2002, 8, 881-890.

4 P. Barai, A. Kumar, P.P. Mukherjee, PLoS ONE, 2016, 11, e0165593.

5 M. Krsmanovic, D. Biswas, H. Ali, A. Kumar, R. Ghosh, A.K. Dickerson, Adv Colloid and Interfac, 2021, 288, 102336.

6 M. F. Moradali, S. Ghods and B. H. Rehm, Front Cell Infect Microbiol, 2017, 7, 39.

7 J. Barbeau, C. Gauthier and P. Payment, Can J Microbiol, 1998, 44, 1019-1028.

8 K. D. Mena and C. P. Gerba, Rev Environ Contam Toxicol, 2009, 201, 71-115.

9 M. M. Ouellet, A. Leduc, C. Nadeau, J. Barbeau and S. J. Charette, Front Microbiol, 2014, 5, 802.

10 C. Gagne-Thivierge, J. Barbeau, R. C. Levesque and S. J. Charette, FEMS Microbiol Lett, 2018, 365.

11 A. T. Vincent, L. Freschi, J. Jeukens, I. Kukavica-Ibrulj, J. G. Emond-Rheault, A. Leduc, B. Boyle, F. Jean-Pierre, M. C. Groleau, E. Deziel, J. Barbeau, S. J. Charette and R. C. Levesque, FEMS Microbiol Ecol, 2017, 93.

12 P. N. Jimenez, G. Koch, J. A. Thompson, K. B. Xavier, R. H. Cool and W. J. Quax, Microbiol Mol Biol Rev, 2012, 76, 46-65.

13 S. Cabrol, A. Olliver, G. B. Pier, A. Andremont and R. Ruimy, J Bacteriol, 2003, 185, 7222-7230.

14 J. H. Hammond, W. P. Hebert, A. Naimie, K. Ray, R. D. Van Gelder, A. DiGiandomenico, P. Lalitha, M. Srinivasan, N. R. Acharya, T. Lietman, D. A. Hogan and M. E. Zegans, mSphere, 2016, 1.

15 M.-C. Groleau, H. Taillefer, A. T. Vincent, P. Constant and E. Déziel, bioRxiv, 2021, DOI: 10.1101/2021.03.25.437011, 2021.2003.2025.437011.

16 J. W. Costerton, Int J Antimicrob Agents, 1999, 11, 217-221; discussion 237-219.

17 F. Abdouchakour, C. Dupont, D. Grau, F. Aujoulat, P. Mournetas, H. Marchandin, S. Parer, P. Gibert, J. Valcarcel and E. Jumas-Bilak, Appl Environ Microbiol, 2015, 81, 7509-7524.

18 P. Thomen, J. Robert, A. Monmeyran, A. F. Bitbol, C. Douarche and N. Henry, PLoS One, 2017, 12, e0175197.

19 J. Azeredo, N. F. Azevedo, R. Briandet, N. Cerca, T. Coenye, A. R. Costa, M. Desvaux, G. Di Bonaventura, M. Hebraud, Z. Jaglic, M. Kacaniova, S. Knochel, A. Lourenco, F. Mergulhao, R. L. Meyer, G. Nychas, M. Simoes, O. Tresse and C. Sternberg, Crit Rev Microbiol, 2017, 43, 313-351.

20 G. A. O'Toole, J Vis Exp, 2011, DOI: 10.3791/2437.

21 H. Ceri, M. E. Olson, C. Stremick, R. R. Read, D. Morck and A. Buret, J Clin Microbiol, 1999, 37, 1771-1776.

22 D. M. Goeres, M. A. Hamilton, N. A. Beck, K. BuckinghamMeyer, J. D. Hilyard, L. R. Loetterle, L. A. Lorenz, D. K. Walker and P. S. Stewart, Nat Protoc, 2009, 4, 783-788.

23 D. M. Goeres, L. R. Loetterle, M. A. Hamilton, R. Murga, D. W. Kirby and R. M. Donlan, Microbiology, 2005, 151, 757-762. 
24 M. Weiss Nielsen, C. Sternberg, S. Molin and B. Regenberg, J Vis Exp, 2011, DOI: 10.3791/2383.

25 M. Pousti, M. P. Zarabadi, M. Abbaszadeh Amirdehi, F. Paquet-Mercier and J. Greener, Analyst, 2018, 144, 68-86.

26 S. Subramanian, R. C. Huiszoon, S. Chu, W. E. Bentley and R. Ghodssi, Biofilm, 2020, 2, 100015.

27 M. Pousti, T. Lefevre, M. A. Amirdehi and J. Greener, Spectrochim Acta A Mol Biomol Spectrosc, 2019, 222, 117163.

28 M. Pousti, M. Joly, P. Roberge, M. A. Amirdehi, A. Bégin-Drolet and J. Greener, Analytical Chemistry, 2018, 90, 14475-14483.

29 24. M. Pousti and J. Greener, Surface Science, 2018, 676, 5660.

30 R. Li, M. Jin, J. Du, M. Li, S. Chen and S. Yang, Front Bioeng Biotechnol, 2020, 8, 957.

31 N. Ceyhan Guvensen, S. Demir and G. Ozdemir, Fresenius Environ. Bull., 2012, 21, 3685-3692.

32 S. Sehar, I. Naz, T. Dasc and S. Ahmed, RSC Adv., 2016, 6, $15112-15120$.

33 P. Stoodley, Z. Lewandowski, J. D. Boyle and H. M. LappinScott, Biotechnol Bioeng, 1999, 65, 83-92.

34 B. Song and L. G. Leff, Microbiol Res, 2006, 161, 355-361.

35 C. K. Stover, X. Q. Pham, A. L. Erwin, S. D. Mizoguchi, P. Warrener, M. J. Hickey, F. S. Brinkman, W. O. Hufnagle, D. J. Kowalik, M. Lagrou, R. L. Garber, L. Goltry, E. Tolentino, S. Westbrock-Wadman, Y. Yuan, L. L. Brody, S. N. Coulter, K. R. Folger, A. Kas, K. Larbig, R. Lim, K. Smith, D. Spencer, G. K. Wong, Z. Wu, I. T. Paulsen, J. Reizer, M. H. Saier, R. E. Hancock, S. Lory and M. V. Olson, Nature, 2000, 406, 959-964.

36 M. Skolimowski, M. W. Nielsen, J. Emneus, S. Molin, R. Taboryski, C. Sternberg, M. Dufva and O. Geschke, Lab Chip, 2010, 10, 2162-2169.

37 L. Ma, M. Conover, H. Lu, M. R. Parsek, K. Bayles and D. J. Wozniak, PLoS Pathog, 2009, 5, e1000354.

38 A. L. Sutlief, H. Valquier-Flynn, C. Wilson, M. Perez, H. Kleinschmidt, B. J. Schofield, E. Delmain, A. E. Holmes and C. D. Wentworth, J Vis Exp, 2019, DOI: 10.3791/58926.

39 O. Lieleg, M. Caldara, R. Baumgärtel and K. Ribbeck, Soft Matter, 2011, 7, 3307-3314.

40 J. Greener, M. Parvinzadeh Gashti, A. Eslami, M. P. Zarabadi and S. M. Taghavi, Biomicrofluidics, 2016, 10, 064107.

41 C. A. Schneider, W. S. Rasband and K. W. Eliceiri, Nat Methods, 2012, 9, 671-675.

42 R. Bakke, R. Kommedal and S. Kalvenes, J Microbiol Methods, 2001, 44, 13-26.

43 F. Paquet-Mercier, M. Parvinzadeh Gashti, J. Bellavance, S. M. Taghavi and J. Greener, Lab Chip, 2016, 16, 4710-4717.

44 J. D. Shrout, D. L. Chopp, C. L. Just, M. Hentzer, M. Givskov and M. R. Parsek, Mol Microbiol, 2006, 62, 1264-1277.

45 P. A. Araújo, J. Malheiro, I. Machado, F. Mergulhão, L. Melo and M. Simões, Journal of Environmental Engineering, 2016, 142, 04016031.

46 M. Simoes, M. O. Pereira, S. Sillankorva, J. Azeredo and M. J. Vieira, Biofouling, 2007, 23, 249-258.

47 A. Crabbe, P. De Boever, R. Van Houdt, H. Moors, M. Mergeay and P. Cornelis, Environ Microbiol, 2008, 10, 2098-2110.

48 M. Parvinzadeh Gashti, J. Bellavance, O. Kroukamp, G. Wolfaardt, S. M. Taghavi and J. Greener, Biomicrofluidics, 2015, 9, 041101.

49 J. Kim, H. S. Kim, S. Han, J. Y. Lee, J. E. Oh, S. Chung and H. D. Park, Lab Chip, 2013, 13, 1846-1849.

50 A. Gjaltema, P. A. Arts, M. C. van Loosdrecht, J. G. Kuenen and J. J. Heijnen, Biotechnol Bioeng, 1994, 44, 194-204.

51 T. Wang, Z. Xu, Y. Li, M. Liang, Z. Wang and P. Hynds, Ecological Indicators, 2016, 71, 598-607.

52 A. Gjaltema, P. A. M. Arts, M. C. M. van Loosdrecht, J. G. Kuenen and J. J. Heijnen, Biotechnology and Bioengineering, 1994, 44, 194-204.
53 F. Ahimou, M. J. Semmens, G. Haugstad and P. J. Novak, Appl Environ Microbiol, 2007, 73, 2905-2910.

54 E. S. Gloag, S. Fabbri, D. J. Wozniak and P. Stoodley, Biofilm, 2020, 2, 100017.

55 H. C. Liou, F. Sabba, A. I. Packman, G. Wells and O. Balogun, Soft Matter, 2019, 15, 575-586.

56 E. Bester, G. M. Wolfaardt, N. B. Aznaveh and J. Greener, Int J Mol Sci, 2013, 14, 21965-21982. 